\title{
PENELOPE, A MONTE CARLO TOOL FOR QUANTITATIVE ELECTRON PROBE MICROANALYSIS
}

\author{
F. Salvat ${ }^{1}$, X. Llovet $^{2}$, J.M. Fernández-Varea ${ }^{1}$ \\ ${ }^{1}$ Facultat de Física (ECM), Universitat de Barcelona. Diagonal 647. 08028 Barcelona. Spain. \\ ${ }^{2}$ Serveis Científico-Tècnics, Univ. Barcelona. Lluís Solé i Sabarís, 1-3. 08028 Barcelona. Spain.
}

PENELOPE (an acronym for PENetration and Energy LOss of Positrons and Electrons) is a general-purpose Monte Carlo tool for the simulation of coupled electron-photon transport in material systems consisting of homogeneous bodies of arbitrary composition [1]. The code covers a wide energy range, from $1 \mathrm{GeV}$ down to $\sim 100 \mathrm{eV}$ and incorporates routines for automatic tracking of particles in quadric geometries and for variance reduction techniques. The complete code system is publicly available from the OECD Nuclear Energy Agency and from the Radiation Shielding Information Computational Center. In this communication we shall focus on the capabilities of the most recent version of PENELOPE (2003) for quantitative electron probe microanalysis, using either the "default" physics models (included in the public version of the code) or more accurate interaction models developed for low-energy applications.

PENELOPE allows the easy simulation of x-ray spectra emitted from homogeneous samples. The code has been validated by comparing simulated x-ray spectra with absolute spectra measured on an electron microprobe, for elements covering the whole periodic system [2]. In general, the agreement between simulation and experiment is very good; the slight discrepancies found are usually attributable to detection artifacts and to inaccuracies in the available data on atomic relaxation.

An advantage of using a general-purpose simulation tool with a robust geometry package is that it can be employed for studying specimens with complex geometrical structures. Thus, PENELOPE has been used to study multilayered structures, grain boundary effects, particulate samples, as well as porous media and rough surfaces.

The physical interaction models implemented in PENELOPE, and in any other general-purpose code, are necessarily approximate. Thus, for example, the photoelectric cross sections pertain to free atoms and, therefore, possible extended x-ray absorption fine structure effects are disregarded. Similarly, the x-ray energies and transition probabilities of inner-shell vacancies are those of free atoms and, consequently, the effect of aggregation on these quantities is neglected. Also the cross sections for certain interaction mechanisms are approximated by means of analytical approximations, which not only allow a reduction in the volume of numerical information needed but also permit the use of numerically robust sampling methods. Nevertheless, the structure of the code has been kept flexible enough to allow the use of alternative, more elaborate physical models when needed.

The accuracy of simulated x-ray spectra is mostly determined by the adopted cross sections for bremsstrahlung emission and inner-shell ionization by electron impact, together with the electron transport model (scattering and slowing down). PENELOPE simulates bremsstrahlung events by using the most accurate differential cross sections (DCS) available, expressed as the 
product of the scaled cross section and the shape function. These DCSs depend on both the energy loss $W$ (i.e. the energy of the emitted photon) and on the direction of emission $\theta$ (relative to the electron direction). An efficient algorithm for random sampling from these DCSs, making allowance for the full correlation between $W$ and $\theta$, has been described by Acosta et al. [3].

The cross sections for ionization of inner ( $\mathrm{K}$ and $\mathrm{L}$ ) shells by electron impact used in PENELOPE were evaluated using the method of virtual quanta. These cross sections are reliable for projectiles with kinetic energies well above the ionization threshold. In our EPMA simulations, we use more accurate cross sections calculated by means of the distorted-wave Born approximation (DWBA) [4]. This approximation accounts for the distortion of the wave function of the projectile caused by the atomic field and for exchange effects in a consistent way. DWBA cross sections are in excellent agreement with experimental data for projectiles with energies from the ionization threshold up to $\sim 100 \mathrm{keV}$.

Elastic scattering is described by using a DCS with a simple analytical form (modified Wentzel), which was determined by fitting the total cross section and the first moments of the DCS calculated by relativistic (Dirac) partial-wave analysis (PWA) for the electrostatic field of the atom. Our analytical DCSs yield results essentially equivalent to those obtained using PWA DCSs whenever the scattering is multiple (i.e. for thick specimens). For thin films it is advisable to use numerical PWA DCS. To date, sampling from PWA DCSs is generally considered as "the method" to describe electron elastic collisions. However, for scattering in gases at energies below $\sim 5 \mathrm{keV}$, the PWA DCS differs systematically from the measured DCS due to the coupling with inelastic channels (absorption); PWA calculations overestimate the DCS at intermediate and large angles. The same effect is expected to occur in condensed matter for low-energy electrons. A relatively simple formulation to account for absorption in elastic scattering is described in ref. [5].

In conclusion, the public version of PENELOPE allows the simulation of x-ray spectra from samples with relatively complex geometries. In most practical cases, the default physical model provides an acceptable description of the measured spectra. The flexibility of the code allows us to implement state-of-the-art interaction data and still keep the easiness of a general-purpose tool.

\section{References}

[1] F. Salvat et al., PENELOPE - A Code System for Monte Carlo Simulation of Electron and Photon Transport. (OECD Nuclear Energy Agency, Issy-les-Moulineaux, 2001).

The PDF file can be downloaded from http://www.nea.fr/lists/penelope.html

[2] X. Llovet et al., J. Appl. Phys. (in press).

[3] E. Acosta, X. Llovet and F. Salvat, Appl. Phys. Lett. 80 (2002) 3228.

[4] S. Segui et al., submitted to Phys. Rev. A

[5] F. Salvat, submitted to Phys. Rev. A 